\title{
Overview of the BioSense 2.0 System Architecture
}

\author{
Kelley Chester* \\ RTI International, Alpharetta, GA, USA
}

\section{Objective}

The objective of this presentation is to provide an overview of the technical architecture of BioSense 2.0.

\section{Introduction}

BioSense 2.0 protects the health of the American people by providing timely insight into the health of communities, regions, and the nation by offering a variety of features to improve data collection, standardization, storage, analysis, and collaboration. BioSense 2.0 is the result of a partnership between the Centers for Disease Control and Prevention (CDC) and the public health community to track the health and well-being of communities across the country. As part of the redesign effort, new fat pipe system architecture has recently been implemented to improve the features and capabilities of the system.

\section{Methods}

Using the latest technology, BioSense 2.0 integrates current health data shared by health departments from a variety of sources to provide insight on the health of communities and the country. By getting more information faster, local, state, and federal public health partners can detect and respond to more outbreaks and health events quicker. From flu outbreaks to car accidents, BioSense 2.0 provides the critical data, information, and tools that public health officials need to better understand and address health problems at the local, state, regional, and national levels. Also, by knowing what is happening across local borders, public health professionals can anticipate potential health problems and respond effectively to protect the health of all people.

This presentation will highlight the architecture of the BioSense 2.0 system, covering the interfaces that data providers use to send data to BioSense and the many methods of viewing the critical public health data contained in the BioSense 2.0 system. Since its inception numerous technical improvements have been made to the BioSense 2.0 system. These changes will be discussed briefly to bring users of the system up to date and to illustrate its benefits and uses to those new to the program.

\section{Conclusions}

Over the past 3 years much has been accomplished during the redesign effort. BioSense 2.0 was launched in November of 2011 and the collaboration between the BioSense program and the public health community has yielded an application based on a user-centered design approach and built on a platform that allows for flexible data sharing across jurisdictions and with partners. The public health community has played a critical role in designing and improving the BioSense 2.0 application, and through continued collaboration, the system will continue to improve.

Innovative features of the BioSense 2.0 application include the use of cloud technology, a novel and flexible data sharing feature; a community-driven approach; enhanced algorithms; and no-cost statistical analysis tools available in the cloud. Each of these features will be discussed during the presentation.

\section{Keywords}

Syndromic Surveillance; Public Health Informatics; Public Health Practice

\author{
*Kelley Chester \\ E-mail: kchester@rti.org
}

